\title{
MHD Mixed Convection Flow over a Permeable Vertical Plate with Buoyancy and Soret Effects
}

\author{
J Prakash *, B Rushi Kumar† and R Sivaraj ${ }^{\ddagger}$
}

\begin{abstract}
This study examines the problem of steady, MHD, mixed convection flow of an incompressible viscous fluid past a semi-infinite vertical permeable plate with slip condition at the boundary layer. The flow field is exposed to the influence of buoyancy, Ohmic heating and Soret effects. The governing equations include the continuity, linear momentum, energy and mass transfer equations which are solved analytically by using perturbation method. The results of this parametric study on the velocity, temperature and concentration distributions are shown graphically and the physical aspects of the problem are highlighted and discussed. The effect of shear stress, rate of heat and mass transfer coefficients at the channel walls are displayed in tables.
\end{abstract}

Keywords: Heat and mass transfer, MHD, mixed convection, buoyancy, Soret effect

* Department of Mathematics, University of Botswana, Private Bag 0022, Gaborone, Botswana, prakashj@mopipi.ub.bw

† Fluid Dynamics Division, SAS, VIT University, Vellore- 632014, Tamil Nadu, India, rushikumar@vit.ac.in

‡ sivaraj.kpm@gmail.com 


\section{Introduction}

MHD heat transfer has gained significance owing to recent advancement of space technology. In recent years, progress has been considerably made in the study of heat and mass transfer in magnetohydrodynamic flows due to its application in many devices, like the MHD power generator and Hall accelerator. We realize the influence of magnetic field from the work of Barletta et al. [1] and Afify [2]. The problem of mixed convection resulting from flow over a heated vertical plate is of considerable theoretical and practical interest. Plenty of different problems involving mixed convection had investigated by Yih [3], Barletle [4], Chin et al. [5], Motsa [6], and Pal and Talukdar [7].

The Soret effect has gained considerable interest in Newtonian convective heat and mass transfer. Such effect is significant when density differences exist in the flow regime. Soret effect is important for intermediate molecular weight gases in coupled heat and mass transfer in binary systems, geophysical systems, often encountered in chemical process engineering and also in highspeed aerodynamics. Kafoussias and Williams [8], Angel et al. [9], Alam and Rahman [10], Abreu et al. [11], and Gaikwad et al. [12] showed the significant of Soret effect in various studies.

Transport phenomena involving the combined influence of thermal and concentration buoyancy are often encountered in many engineering systems and natural environments. There are many applications of such transport processes in the industry, notably in chemical distilleries, heat exchangers, solar energy collectors and thermal protection systems. The interaction of buoyancy has increased greatly during the last decade due to its importance in many practical applications. The effect of buoyancy was analyzed for different problems by El-Alimi et al. [13], Shateyi [14], and Elzubier et al. [15]. Ohmic heating is a developing technology with considerable potential for the food industry. The main advantages of Ohmic processing are the rapid and relatively uniform heating achieved, together with the lower capital cost compared to other 52 
electro heating methods such as microwave and radio frequency heating. The potential applications of Ohmic heating technique in food industry are very wide, for example blanching, evaporation, dehydration and pasteurization. In view of these applications AboEldahab and El-Aziz [16], Osalusi and Harris [17], and Pal and Talukdar [18] investigated problems involving the Ohmic dissipation effect. It is more realistic to include Ohmic effect in order to explore the impact of the magnetic field on the thermal transport in the boundary layer.

Motivated by the above referenced works and the numerous possible industrial applications of engineering fields, it is of paramount interest in this study to analyze the steady magnetohydrodynamic mixed convective heat and mass transfer problem of a viscous incompressible fluid flow with the influence of buoyancy, Ohmic heating, viscous dissipation, heat absorption parameter and Soret effect. We solve this problem analytically by using perturbation method and the effects of all significant parameters are studied analytically. We illustrate the influence of various flow parameters on the velocity, temperature, concentration skin friction coefficient, Nusselt number and Sherwood number distributions through graphs. The rest of the paper is structured as follows: we present the governing equations in Section 2. We proceed in Section 3 to solve the set of equations of the viscous incompressible fluid by using perturbation method. Section 4 provides a discussion of results and we present conclusions in Section 5.

\section{Formulation of the Problem}

We consider the steady two-dimensional flow of an incompressible, viscous, electrically conducting and heat-absorbing fluid past a semi-infinite vertical permeable plate subject to slip boundary condition at the interface of fluid layers. A uniform transverse magnetic field of magnitude $B_{0}$ is applied in the presence of radiation and concentration buoyancy effects in the direction of $y$-axis. Also, viscous dissipative and Ohmic dissipative effects are present with Soret effect. 


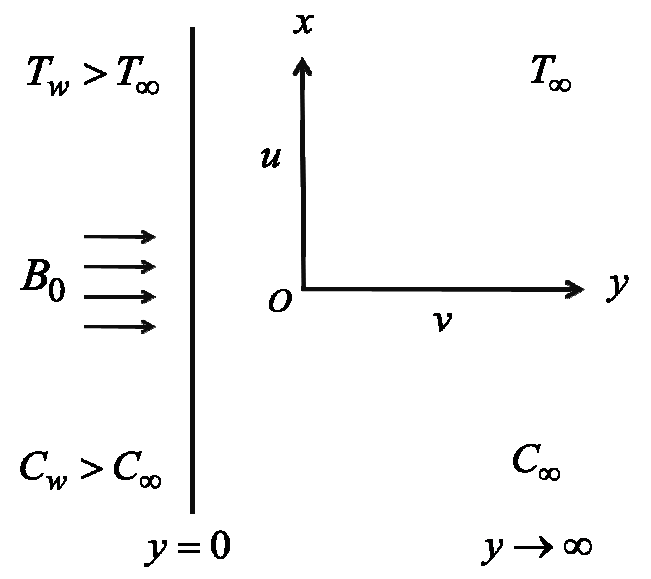

Fig. 1- Flow geometry of the problem

The wall $y=0$ is maintained at a constant temperature $T_{w}$ and concentration $C_{w}$, higher than the ambient temperature $T_{\infty}$ and ambient concentration $C_{\infty}$, respectively. Rest of properties of the fluid are assumed to be constant.

The governing equations for this investigation are based on the balances of mass, linear momentum, energy and concentration species. Taking into consideration these assumptions, the equations that describe the physical situation can be represented as follows:

$$
\begin{gathered}
\frac{\partial u}{\partial x}+\frac{\partial v}{\partial y}=0 \\
\left(u \frac{\partial u}{\partial x}+v \frac{\partial u}{\partial y}\right)=-\frac{1}{\rho} \frac{\partial p}{\partial x}+v\left(\frac{\partial^{2} u}{\partial x^{2}}+\frac{\partial^{2} u}{\partial y^{2}}\right)-\frac{\sigma B_{0}^{2}}{\rho} u \\
+g \beta_{T}\left(T-T_{\infty}\right)+g \beta_{C}\left(C-C_{\infty}\right) \\
\left(u \frac{\partial v}{\partial x}+v \frac{\partial v}{\partial y}\right)=-\frac{1}{\rho} \frac{\partial p}{\partial y}+v\left(\frac{\partial^{2} v}{\partial x^{2}}+\frac{\partial^{2} v}{\partial y^{2}}\right)
\end{gathered}
$$




$$
\begin{aligned}
&\left(u \frac{\partial T}{\partial x}+v \frac{\partial T}{\partial y}\right)= \frac{K}{\rho C_{p}}\left(\frac{\partial^{2} T}{\partial x^{2}}+\frac{\partial^{2} T}{\partial y^{2}}\right)-\frac{Q\left(T-T_{\infty}\right)}{\rho C_{p}} \\
&+\frac{v}{C_{p}}\left(\frac{\partial u}{\partial x}+\frac{\partial u}{\partial y}\right)^{2}+\frac{\sigma B_{0}^{2} u^{2}}{\rho C_{p}} \\
&\left(u \frac{\partial C}{\partial x}+v \frac{\partial C}{\partial y}\right)=D\left(\frac{\partial^{2} C}{\partial x^{2}}+\frac{\partial^{2} C}{\partial y^{2}}\right)+\frac{D K_{T}}{\bar{T}}\left(\frac{\partial^{2} T}{\partial x^{2}}+\frac{\partial^{2} T}{\partial y^{2}}\right)
\end{aligned}
$$

The boundary conditions of the problem are

$$
\begin{aligned}
& u=u_{\text {slip }}=\frac{\sqrt{k}}{\gamma_{1}} \frac{\partial u}{\partial y}, \quad T=T_{w}, \quad C=C_{w} \quad \text { at } \quad y=0 \\
& u \rightarrow u_{\infty}=0, \quad T_{w} \rightarrow T_{\infty}, \quad C_{w} \rightarrow C_{\infty} \quad \text { as } \quad y \rightarrow \infty
\end{aligned}
$$

where $x$ and $y$ are the dimensional distances along and perpendicular to the plate, respectively. $u$ and $v$ are the components of dimensional velocity $u$ along $x$ and $y$ directions, respectively. $g$ is the gravitational acceleration, $p$ is the pressure, $B_{0}$ is the magnetic field coefficient, $\sigma$ is the magnetic permeability of the fluid, $\rho$ is the density, $\mu$ is viscosity of the fluid, $v$ is the kinematic viscosity, $\beta_{T}$ and $\beta_{C}$ are the thermal and concentration expansion coefficients, respectively. $T$ is the dimensional temperature of the fluid, $C_{p}$ is the specific heat of constant pressure, $K$ is the thermal conductivity, $Q$ is the dimensional heat absorption coefficient, $C$ is the dimensional concentration, $D$ is the molecular diffusivity, $K_{T}$ is the thermal diffusion ratio, $\bar{T}$ is the mean temperature of $T_{w}$ and $T_{\infty}, k$ is the permeability of the porous medium and $\gamma_{1}$ is the dimensional porous parameter. The fourth and fifth terms on RHS of the momentum equation (2) denote the thermal and solutal buoyancy effects, respectively. The third and fourth term on the RHS of equation (4) denote the inclusion of viscous dissipation and Ohmic dissipation effects, respectively. 
Since the motion is two dimensional and length of the plate is large enough so all the physical variables are independent of $x$-axis. Therefore,

$$
\frac{\partial u}{\partial x}=0
$$

We consider that the suction velocity at the plate surface is a constant and the suction velocity takes the following form

$$
v=-V_{0}
$$

where $V_{0}$ is a scale of suction velocity which has non-zero positive constant.

Outside the boundary layer, Eq. (2) gives

$$
-\frac{1}{\rho} \frac{d p}{d x}=A
$$

where $(A<<1)$ is the pressure gradient and Eq. (3) reduces to

$$
\frac{d p}{d y}=0
$$

so that the pressure $p$ is independent of $y$.

Introducing the following non-dimensional quantities

$$
U=\frac{u}{U_{0}}, \quad V=\frac{v}{V_{0}}, \quad Y=\frac{V_{0} y}{v}, \quad T=\frac{\theta-T_{\infty}}{T_{w}-T_{\infty}}, \quad C=\frac{\phi-C_{\infty}}{C_{w}-C_{\infty}}
$$

In view of the above non-dimensional variables, the basic field of Eqs. (2), (4) and (5) can be expressed in non-dimensional form as

$$
\begin{gathered}
\frac{d^{2} U}{d Y^{2}}+\frac{d U}{d Y}-M^{2} U+G r \theta+G c \phi+A=0 \\
\frac{d^{2} \theta}{d Y^{2}}+\operatorname{Pr} \frac{d \theta}{d Y}-\operatorname{Pr} \alpha \theta+\operatorname{Pr} E\left(\frac{d U}{d Y}\right)^{2}+\operatorname{Pr}^{2} E U^{2}=0
\end{gathered}
$$




$$
\frac{d^{2} \phi}{d Y^{2}}+S c \frac{d \phi}{d Y}+\operatorname{Sr}\left(\frac{d^{2} \theta}{d Y^{2}}\right)=0
$$

Together with boundary conditions

$$
\begin{gathered}
U=U_{\text {slip }}=\gamma \frac{d U}{d Y}, \theta=1, \phi=1 \text { at } Y=0 \\
U \rightarrow 0, \theta \rightarrow 0, \phi \rightarrow 0 \text { as } Y \rightarrow \infty
\end{gathered}
$$

where $G r$ is the Grashof number, $G c$ is the solutal Grashof number, $M^{2}$ is the magnetic field parameter, $\operatorname{Pr}$ is the Prandtl number, $\alpha$ is the heat absorption parameter, $S c$ is the Schmidt number, $E$ is the Eckert number, $S r$ Soret number and $\gamma_{1}$ dimensional porous permeability parameter which are defined as follows:

$$
\begin{aligned}
& G r=\frac{v g \beta_{T}\left(T_{w}-T \infty\right)}{U_{0} V_{0}^{2}}, \quad G c=\frac{v g \beta_{C}\left(C_{w}-C \infty\right)}{U_{0} V_{0}^{2}}, \quad M^{2}=\frac{\sigma B_{0}^{2} v}{\rho V_{0}^{2}}, \\
& \operatorname{Pr}=\frac{\mu C_{p}}{K}, \quad \alpha=\frac{Q v}{\rho C_{p} V_{0}^{2}}, \quad S c=\frac{v}{D}, \quad E=\frac{U_{0}^{2}}{C_{p}\left(T_{w}-T \infty\right)}, \\
& S r=\frac{K_{T}\left(T_{w}-T \infty\right)}{\bar{T}\left(C_{w}-C \infty\right)} \text { and } \gamma=\frac{\sqrt{k}}{\gamma_{1}} \frac{U_{0} V_{0}}{v} .
\end{aligned}
$$

The Eckert number is always less than unity since the flow due to the Joules dissipation is supper imposed on the main flow so that we consider as

$$
E<<1
$$

\section{Method of Solution}

The set of partial differential Eqs. (13) - (15) cannot be solved in closed-form. However, it can be solved analytically after these equations are reduced to a set of ordinary differential equations in dimensionless form which can be done by representing the velocity $U$, temperature $\theta$ and concentration $\phi$ in terms of power of Eckert number $\mathrm{E}$ as follows: 


$$
\begin{gathered}
U(Y)=f_{0}(Y)+E f_{1}(Y)+o\left(E^{2}\right) \\
\theta(Y)=\theta_{0}(Y)+E \theta_{1}(Y)+o\left(E^{2}\right) \\
\phi(Y)=\phi_{0}(Y)+E \phi_{1}(Y)+o\left(E^{2}\right)
\end{gathered}
$$

Substituting (20)-(22) into Eqs. (13)-(15) and equating the corresponding terms of the equations, neglecting the higher order of $o\left(E^{2}\right)$ and simplifying to get the following pairs of equations for $f_{0}, \theta_{0}, \phi_{0}$ and $f_{1}, \theta_{1}, \phi_{1}$

$$
\begin{gathered}
f_{0}^{\prime \prime}+f_{0}^{\prime}-M^{2} f_{0}=-\left(A+G r \theta_{0}+G c \phi_{0}\right) \\
f_{1}^{\prime \prime}+f_{1}^{\prime}-M^{2} f_{1}=-\left(\operatorname{Gr} \theta_{1}+G c \phi_{1}\right) \\
\theta_{0}^{\prime \prime}+\operatorname{Pr} \theta_{0}^{\prime}-\operatorname{Pr} \alpha \theta_{0}=0 \\
\theta_{1}^{\prime \prime}+\operatorname{Pr} \theta_{1}^{\prime}-\operatorname{Pr} \alpha \theta_{1}=-\left(\operatorname{Pr}\left(U_{0}^{\prime}\right)^{2}-\operatorname{PrM} U_{0}^{2}\right) \\
\phi_{0}^{\prime \prime}+\operatorname{Sc} \phi_{0}^{\prime}=-\operatorname{Sr} \theta_{0}^{\prime \prime} \\
\phi_{1}^{\prime \prime}+\operatorname{Sc} \phi_{1}^{\prime}=-\operatorname{Sr} \theta_{1}^{\prime \prime}
\end{gathered}
$$

where the prime denotes ordinary differentiation with respect to $Y$ . The corresponding boundary conditions are

$$
\begin{aligned}
& f_{0}=\gamma_{1} f_{0}^{\prime}, f_{1}=\gamma_{1} f_{1}^{\prime}, \theta_{0}=1, \theta_{1}=0, \phi_{0}=1, \phi_{1}=0 \text { at } Y=0 \\
& f_{0} \rightarrow 0, f_{1} \rightarrow 0, \theta_{0} \rightarrow 0, \theta_{1} \rightarrow 0, \phi_{0} \rightarrow 0, \phi_{1} \rightarrow 0 \text { as } Y \rightarrow \infty
\end{aligned}
$$

The solutions of Eqs. (23) - (28) with the help of boundary conditions (29) and (30), are

$$
f_{0}=A_{3} e^{-\beta_{1} Y}+A_{4} e^{-\beta_{2} Y}+A_{6} e^{-\beta_{3} Y}+A_{5}
$$




$$
\begin{aligned}
f_{1}= & A_{30} e^{-\beta_{1} Y}+A_{31} e^{-\beta_{2} Y}+A_{41} e^{-\beta_{3} Y}+A_{33} e^{-2 \beta_{1} Y}+A_{34} e^{-2 \beta_{2} Y}+A_{35} e^{-2 \beta_{3} Y} \\
& +A_{36} e^{-\left(\beta_{1}+\beta_{2}\right) Y}+A_{37} e^{-\left(\beta_{2}+\beta_{3}\right) Y}+A_{38} e^{-\left(\beta_{1}+\beta_{3}\right) Y} \\
\theta_{0}=e^{-\beta_{1} Y} & \\
\theta_{1}= & A_{17} e^{-\beta_{1} Y}+A_{8} e^{-\beta_{2} Y}+A_{9} e^{-\beta_{3} Y}+A_{10} e^{-2 \beta_{1} Y}+A_{11} e^{-2 \beta_{2} Y}+A_{12} e^{-2 \beta_{3} Y} \\
& +A_{13} e^{-\left(\beta_{1}+\beta_{2}\right) Y}+A_{14} e^{-\left(\beta_{2}+\beta_{3}\right) Y}+A_{15} e^{-\left(\beta_{1}+\beta_{3}\right) Y}+A_{16} \\
& \phi_{0}=A_{1} e^{-\beta_{1} Y}+A_{2} e^{-\beta_{2} Y} \\
\phi_{1}= & A_{19} e^{-\beta_{1} Y}+A_{29} e^{-\beta_{2} Y}+A_{21} e^{-\beta_{3} Y}+A_{22} e^{-2 \beta_{1} Y}+A_{23} e^{-2 \beta_{2} Y}+A_{24} e^{-2 \beta_{3} Y} \\
& +A_{25} e^{-\left(\beta_{1}+\beta_{2}\right) Y}+A_{26} e^{-\left(\beta_{2}+\beta_{3}\right) Y}+A_{27} e^{-\left(\beta_{1}+\beta_{3}\right) Y}
\end{aligned}
$$

where $A^{\prime}$ s and $\beta$ 's are given in Appendix.

Substituting the above solutions (31) - (36) in (20) - (22), we get the final form of velocity, temperature and concentration distributions in the boundary layer as follows:

$$
\begin{aligned}
U(Y)= & A_{3} e^{-\beta_{1} Y}+A_{4} e^{-\beta_{2} Y}+A_{6} e^{-\beta_{3} Y}+A_{5} \\
+ & E\left[\begin{array}{l}
A_{30} e^{-\beta_{1} Y}+A_{31} e^{-\beta_{2} Y}+A_{41} e^{-\beta_{3} Y} \\
+A_{33} e^{-2 \beta_{1} Y}+A_{34} e^{-2 \beta_{2} Y}+A_{35} e^{-2 \beta_{3} Y} \\
+A_{36} e^{-\left(\beta_{1}+\beta_{2}\right) Y}+A_{37} e^{-\left(\beta_{2}+\beta_{3}\right) Y}+A_{38} e^{-\left(\beta_{1}+\beta_{3}\right) Y}
\end{array}\right] \\
\theta(Y)= & e^{-\beta_{1} Y} \\
& +E\left[\begin{array}{l}
A_{17} e^{-\beta_{1} Y}+A_{8} e^{-\beta_{2} Y}+A_{9} e^{-\beta_{3} Y}+A_{10} e^{-2 \beta_{1} Y} \\
+A_{11} e^{-2 \beta_{2} Y}+A_{12} e^{-2 \beta_{3} Y}+A_{13} e^{-\left(\beta_{1}+\beta_{2}\right) Y} \\
+A_{14} e^{-\left(\beta_{2}+\beta_{3}\right) Y}+A_{15} e^{-\left(\beta_{1}+\beta_{3}\right) Y}+A_{16}
\end{array}\right] \\
\phi(Y)= & A_{1} e^{-\beta_{1} Y}+\left(1-A_{1}\right) e^{-\beta_{2} Y} \\
+ & {\left[\begin{array}{l}
A_{19} e^{-\beta_{1} Y}+A_{29} e^{-\beta_{2} Y}+A_{21} e^{-\beta_{3} Y} \\
+A_{22} e^{-2 \beta_{1} Y}+A_{23} e^{-2 \beta_{2} Y}+A_{24} e^{-2 \beta_{3} Y} \\
+A_{25} e^{-\left(\beta_{1}+\beta_{2}\right) Y}+A_{26} e^{-\left(\beta_{2}+\beta_{3}\right) Y}+A_{27} e^{-\left(\beta_{1}+\beta_{3}\right) Y}
\end{array}\right] }
\end{aligned}
$$


The physical quantities of interest are the wall shear stress $\tau_{w}$ is given by

$$
\tau_{w}=\left.\mu \frac{\partial u}{\partial y}\right|_{y=0}=\left.\frac{U_{0} V_{0}}{\rho} \frac{\partial U}{\partial Y}\right|_{Y=0}
$$

Therefore, the local skin friction factor $\tau$ is given by

$$
\begin{gathered}
\tau=\frac{\tau_{w} \rho}{U_{0} V_{0}}=U^{\prime}(0) \\
\tau=-\left[A_{2} \beta_{1}+A_{3} \beta_{2}+A_{4} \beta_{3}\right] \\
-E\left[\begin{array}{l}
A_{30} \beta_{1}+A_{31} \beta_{2}+A_{32} \beta_{3} \\
+2 A_{33} \beta_{1}+2 A_{34} \beta_{2}+2 A_{35} \beta_{3} \\
+A_{36}\left(\beta_{1}+\beta_{2}\right)+A_{37}\left(\beta_{2}+\beta_{3}\right)+A_{38}\left(\beta_{1}+\beta_{3}\right)
\end{array}\right]
\end{gathered}
$$

The local surface heat flux is given by

$$
q_{w}=-\left.K \frac{\partial T}{\partial y}\right|_{y=0}=\left.\frac{-K\left(T_{w}-T_{\infty}\right) V_{0}}{v} \frac{\partial \theta}{\partial Y}\right|_{Y=0}
$$

where $K$ is the effective thermal conductivity.

The local Nusselt number $N u_{x}=-q_{w} /\left(T_{w}-T_{\infty}\right)$ can be written as

$$
N u=\frac{N u_{x}}{R e_{x}}=\theta^{\prime}(0)
$$

$$
\begin{aligned}
N u=- & \beta_{1} \\
& -E\left[\begin{array}{l}
A_{17} \beta_{1}+A_{8} \beta_{2}+A_{9} \beta_{3} \\
+2 A_{10} \beta_{1}+2 A_{11} \beta_{2}+2 A_{12} \beta_{3} \\
+A_{13}\left(\beta_{1}+\beta_{2}\right)+A_{14}\left(\beta_{2}+\beta_{3}\right)+A_{15}\left(\beta_{1}+\beta_{3}\right)
\end{array}\right]
\end{aligned}
$$

The local surface mass flux is given by

$$
S h=\frac{S h_{x}}{R e_{x}}=\left.\frac{\partial \phi}{\partial Y}\right|_{Y=0}=\phi^{\prime}(0)
$$




$$
\begin{aligned}
S h= & -\left[A_{1} \beta_{1}+A_{2} \beta_{2}\right] \\
& -E\left[\begin{array}{l}
A_{19} \beta_{1}+A_{29} \beta_{2}+A_{21} \beta_{3} \\
+2 A_{22} \beta_{1}+2 A_{23} \beta_{2}+2 A_{24} \beta_{3} \\
+A_{25}\left(\beta_{1}+\beta_{2}\right)+A_{26}\left(\beta_{2}+\beta_{3}\right)+A_{27}\left(\beta_{1}+\beta_{3}\right)
\end{array}\right]
\end{aligned}
$$

where $\operatorname{Re}_{x}=V_{0} K / v$ is the local Reynolds number.

\section{Result and Discussion}

Graphical representation of results is very useful to discuss the physical features presented by the solutions. In order to get a physical insight of this problem, factors such as velocity, temperature, concentration, Skin friction, Nusselt number and Sherwood number have been discussed by assigning numerical values to various parameters obtained in the mathematical formulation of the problem and the results are graphically shown in Figures 2-19.

The impressions of a transverse magnetic field to an electrically conducting fluid gives rise to a resistive type force called the Lorentz force. This force has the tendency to slow down the motion of the fluid. Application of a magnetic field moving with the free stream has the tendency to induce a motive force which decreases the motion of the fluid and which is displayed in Fig. 2. The increments of porous permeability parameter are used to support the velocity profile and it is shown in Fig. 3. Usually the Grashof number and solutal Grashof number boost the fluid velocity. The effect of increasing Grashof number and solutal Grashof number is used to increase the velocity field as expected and it is shown in Fig. 4 and Fig. 5, respectively. Figure 6 indicates that increasing heat absorption parameter suppresses the velocity significantly. The velocity field shrinks for the rise of Prandtl number which is shown in Fig. 7. 


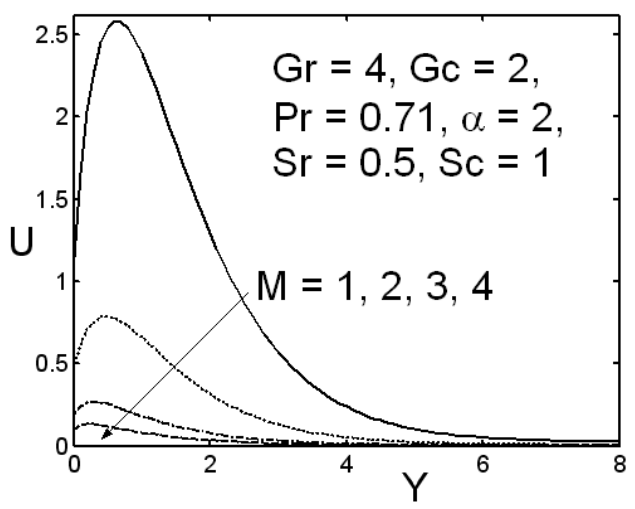

Fig. 2- Effect of magneic field invelocity distribution

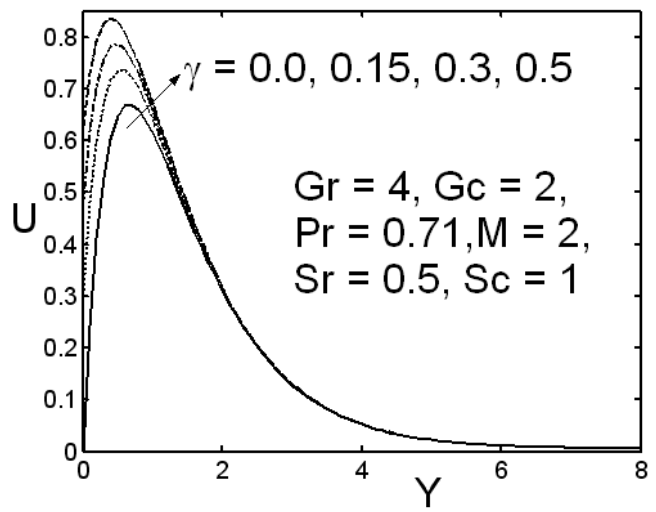

Fig. 3- Effect of porous permiability in velocity distribution

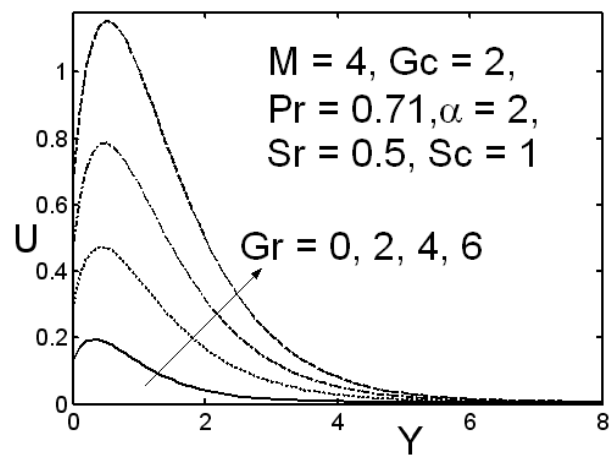

Fig. 4- Effect of thermal Grashof number in velocity distribution 


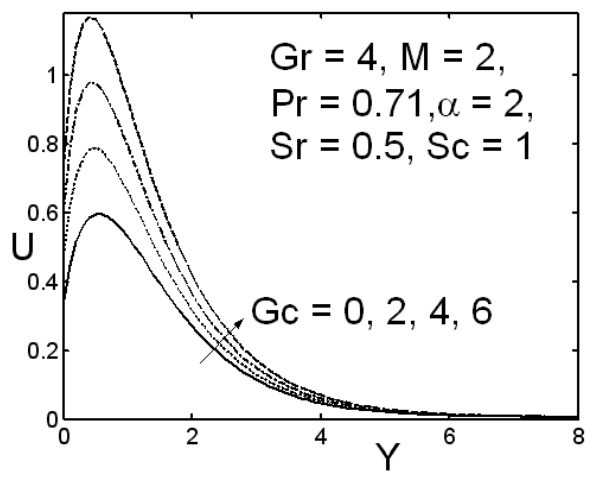

Fig. 5- Effect of solutal Grashof number in velocity distribution

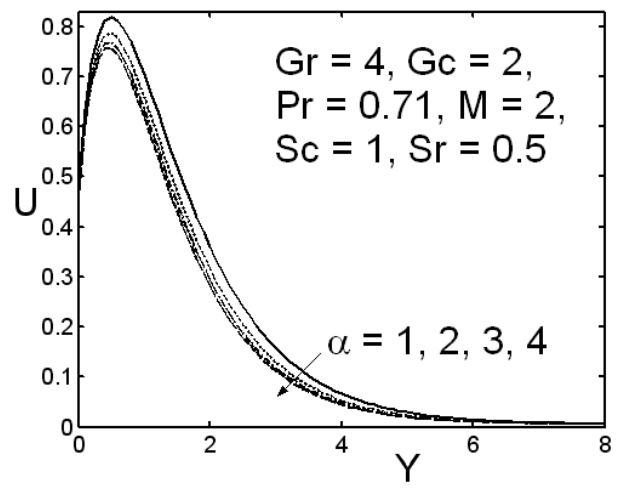

Fig. 6- Effect of heat absorbtion parameter in velocity distribution

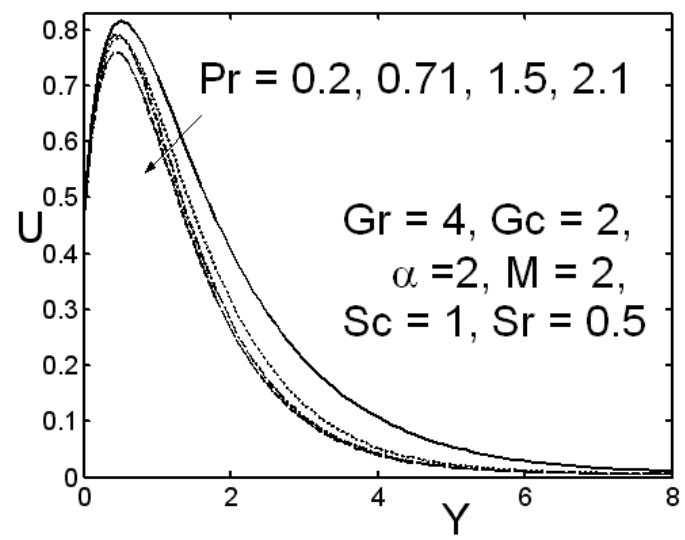

Fig. 7- Effect of Prandtl number invelocity distribution 


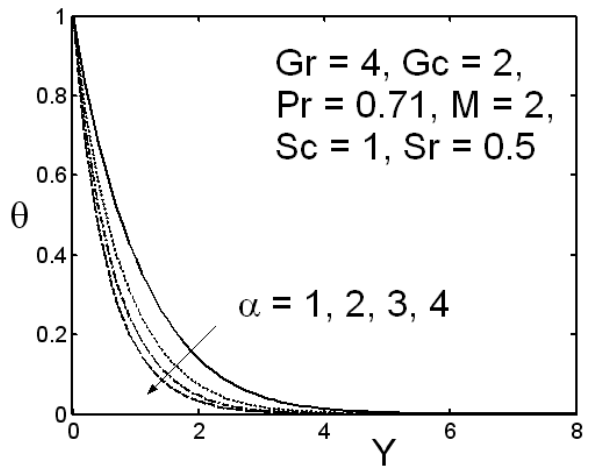

Fig. 8- Effect of heat absorbtion parameter in temperature distribution

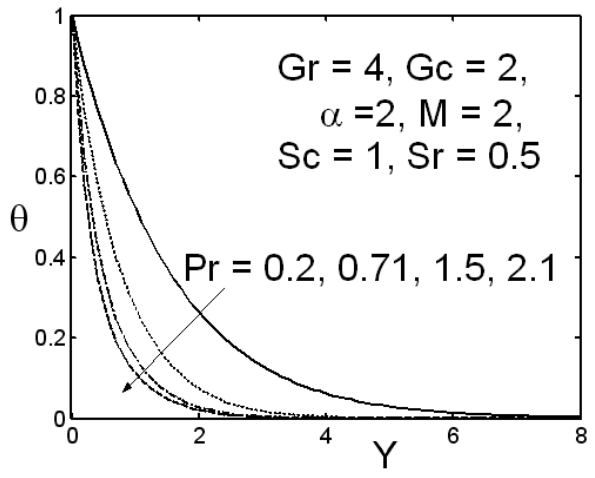

Fig. 9- Effect of Prandtl number in temperature distribution

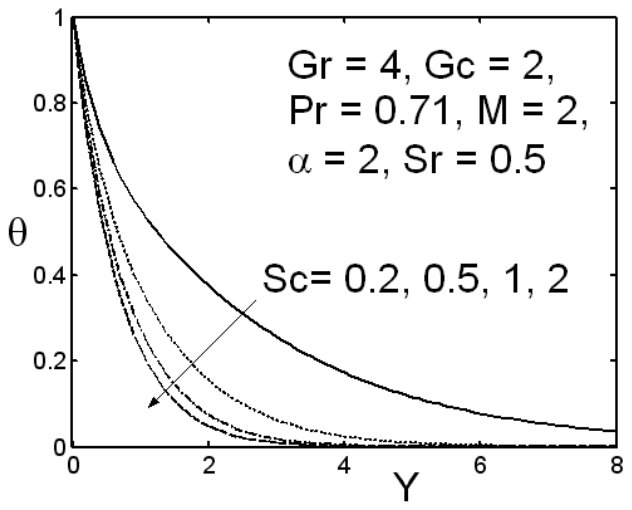

Fig. 10- Effect of Schmidt number in temperature distribution 


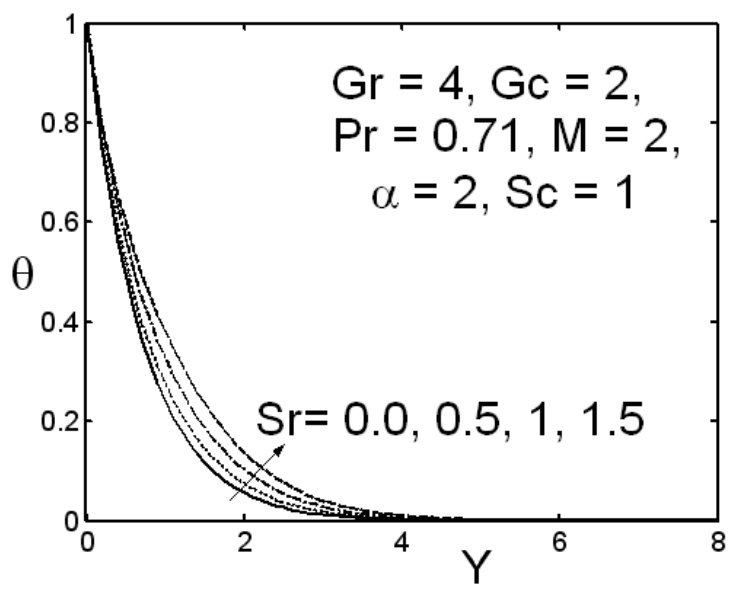

Fig. 11- Effect of Soret number in temperature distribution

It is well known that the heat absorption (i.e. $\alpha<0$ ) causes the fluid temperature to decrease because which has the tendency to decrease the thermal buoyancy effects. Therefore the increments of heat source parameter diminish the heat transfer and we illustrate this in Fig. 8. The increase of Prandtl numbers is equivalent to increasing the thermal conductivities, and therefore heat is able to diffuse away from the heated plate more rapidly. So increase in the Prandtl number results a decrease of fluid temperature which is presented in Fig. 9. Figure 10 illustrates that the heat transfer falls off for the rise of Schmidt number. An increase in the Soret number is used to increase the boundary layer thickness. Therefore the heat dispersal will be reduced and heat transfer increases for increasing the Soret number which is plotted in Fig. 11.

An increase in Schmidt number decreases molecular diffusion $D$. Hence, the mass transfer of the species is higher for small values of Schmidt number and lower for larger values of Schmidt number and we plot this in Fig. 12. The concentration increases for increasing Soret number and it is observed from Fig. 13. 


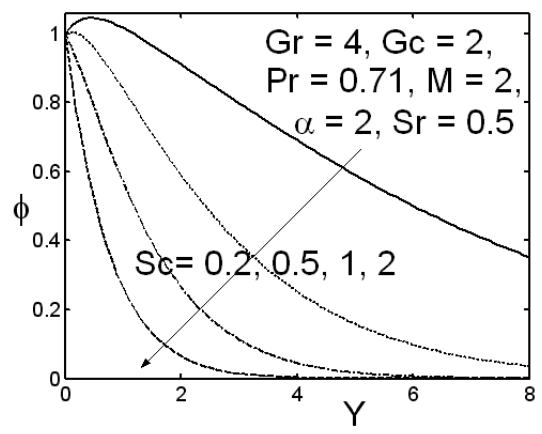

Fig. 12- Effect of Schmidt number in concentration distribution

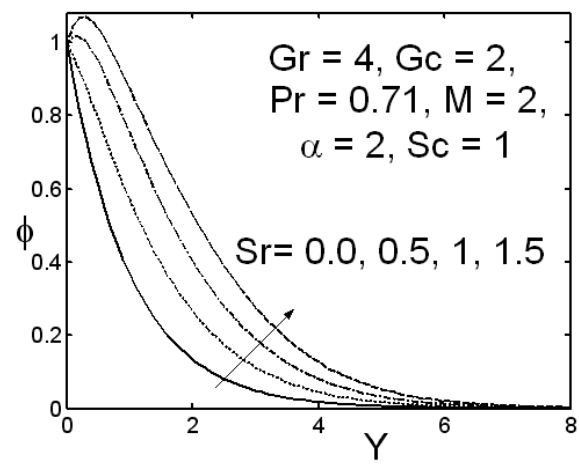

Fig. 13- Effect of Soret number in concentration distribution

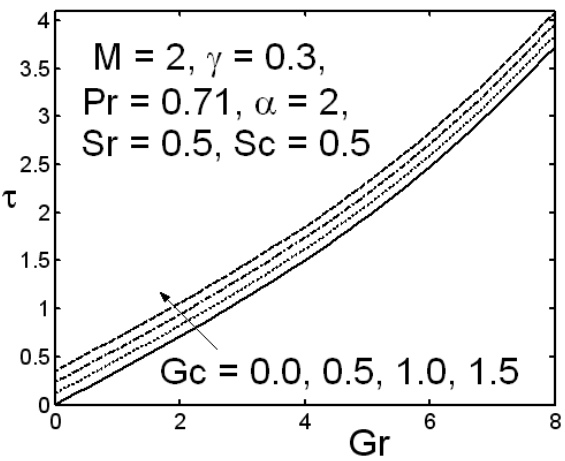

Fig. 14- Effect of solutal Grashof number in skin friction distribution 


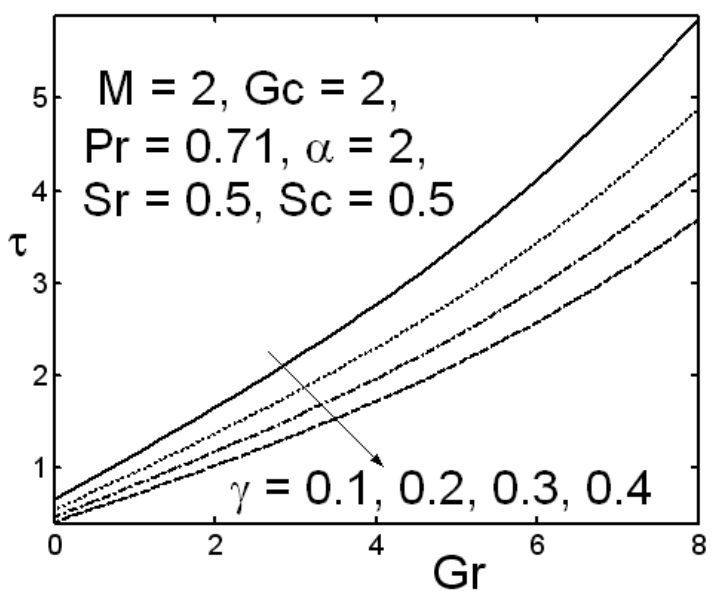

Fig. 15- Effect of porous permiability parameter in skin friction distribution

We present the variation of the local skin friction co-efficient $\tau$ against the thermal Grashof number for various values of $\gamma$ and Gc. Figure 14 shows that the increments of mass Grashof number used to support the rate of fluid velocity. It is noticed from Fig. 15 that value of the shearing stress falls rapidly for the rise of porous permeability parameter. The Nusselt number $N u$ against the magnetic field parameter $M$ for various values of $\alpha$ and $\operatorname{Pr}$ are graphically displayed. The Nusselt number decreases monotonically for increasing the value of heat absorption parameter and Prandtl number which are showed through The Fig. 16 and Fig. 17, respectively. The Sherwood number $S h$ against the magnetic field parameter $M$ for various values of $S c$ and $S r$ is presented graphically. Figure 18 illustrates that an increase in Schmidt number result in fall off rate of mass transfer. The Sherwood number profiles increase with an increase in Soret number and the curves could be seen in Fig. 19. Throughout the computations we employ $E=0.2$ and $A=0.02$. It is observed that the agreement with the theoretical solution of all profiles is excellent. 


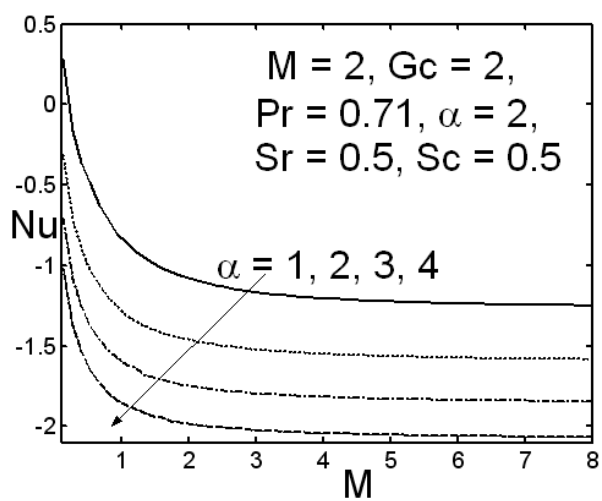

Fig. 16- Effect of heat absorption parameter in Nusselt number distribution

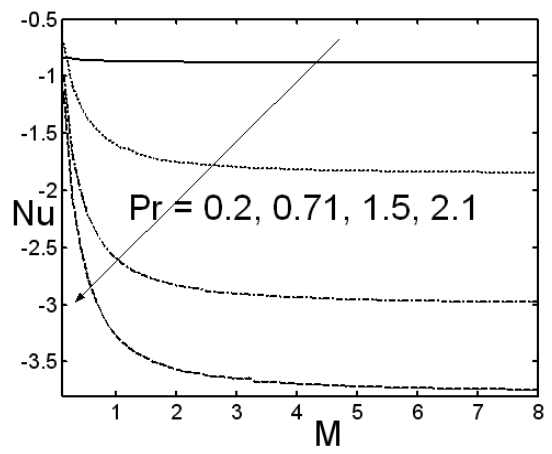

Fig. 17- Effect of Prandtl number in Nusselt number distribution

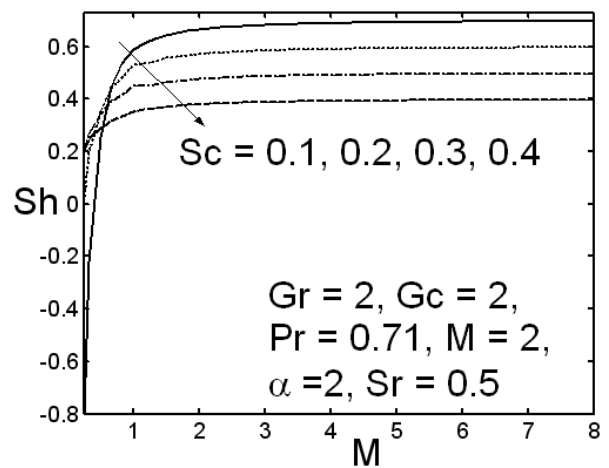

Fig. 18- Effect of Schmidt number in Sherwood number distribution 


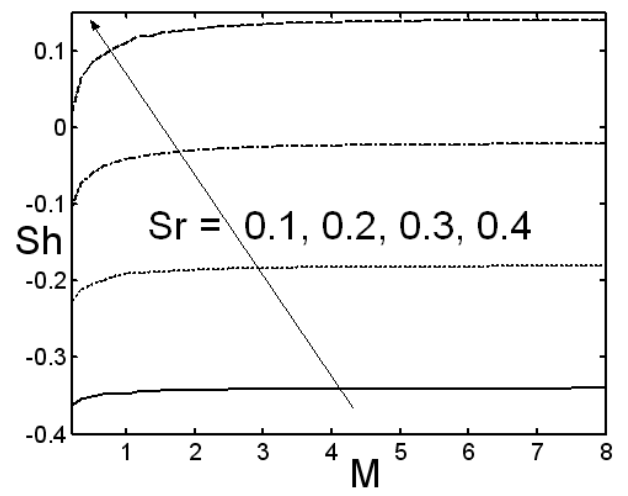

Fig. 19- Effect of Soret number in Sherwood number distribution

Tables 1 to 4 are presented to show the influence of $G r, G c, \alpha$ and $S c$ in skin friction, Nusselt number and Sherwood number distributions. Tables $1 \& 2$ show that an increase in the thermal and mass Grashof numbers increases the skin friction and Nusselt number where as it decreases the Sherwood number. Table 3 displays that the skin friction and Nusselt number distributions decrease for increasing the heat absorption. The Sherwood number increases with an increase in the heat absorption parameter. Table 4 displays that an increase in the Soret number increases the skin friction, Nusselt number and Sherwood number distributions.

TABLE 1: Effect of $G r$ in $\tau_{0}, N u_{0} \& S h_{0}$

\begin{tabular}{|c|c|c|c|}
\hline$G r$ & $\tau_{0}$ & $N u_{0}$ & $S h_{0}$ \\
\hline \hline 0.0000 & 0.4746 & -1.5901 & 0.1950 \\
\hline \hline 1.0000 & 0.8199 & -1.5453 & 0.1722 \\
\hline \hline 2.0000 & 1.1772 & -1.4613 & 0.1299 \\
\hline \hline 3.0000 & 1.5578 & -1.3381 & 0.0679 \\
\hline
\end{tabular}


TABLE 2: Effect of $G c$ in $\tau_{0}, N u_{0} \& S h_{0}$

\begin{tabular}{|c|c|c|c|}
\hline$G c$ & $\tau_{0}$ & $N u_{0}$ & $S h_{0}$ \\
\hline 0.0000 & 0.7108 & -1.5170 & 0.1577 \\
\hline 0.5000 & 0.8277 & -1.5048 & 0.1516 \\
\hline 1.0000 & 0.9445 & -1.4914 & 0.1450 \\
\hline 1.5000 & 1.0610 & -1.4769 & 0.1377 \\
\hline
\end{tabular}

TABLE 3: Effect of $\alpha$ in $\tau_{0}, N u_{0} \& S h_{0}$

\begin{tabular}{|c|c|c|c|}
\hline$\alpha$ & $\tau_{0}$ & $N u_{0}$ & $S h_{0}$ \\
\hline
\end{tabular}

\begin{tabular}{|l|l|l|l|}
\hline 1.0000 & 1.2105 & -1.0796 & -0.0617 \\
\hline
\end{tabular}

\begin{tabular}{|l|l|l|l|}
\hline 3.0000 & 1.1568 & -1.7460 & 0.2725 \\
\hline
\end{tabular}

\begin{tabular}{|l|l|l|l|}
\hline 4.0000 & 1.1422 & -1.9825 & 0.3908 \\
\hline
\end{tabular}

TABLE 4: Effect of $S r$ in $\tau_{0}, N u_{0} \& S h_{0}$

\begin{tabular}{|c|c|c|c|}
\hline$S r$ & $\tau_{0}$ & $N u_{0}$ & $S h_{0}$ \\
\hline 0.0000 & 1.0370 & -1.5140 & -0.6000 \\
\hline 0.5000 & 1.1772 & -1.4613 & 0.1299 \\
\hline 1.0000 & 1.3101 & -1.3942 & 0.7921 \\
\hline 1.5000 & 1.4321 & -1.3130 & 1.3652 \\
\hline
\end{tabular}




\section{Conclusions}

The mathematical modeling for the steady MHD mixed convective heat and mass transfer boundary layer slip flow in permeable vertical plates with the influence of buoyancy, Ohmic heating and Soret effect has been analyzed. An analytical solution is obtained for this problem by using perturbation method and numerical results are presented in graphs and tables. The numerical calculation results satisfy the boundary layer conditions mathematically. The effects of various significant parameters on this study are analyzed. The key observations of the present study are listed below.

i. An increase in the magnetic field diminishes the fluid velocity

ii. The rise of thermal and mass buoyancy effects and porous permeability parameter are used to support the fluid velocity

iii. An increase in heat absorption parameter and Prandtl number is to diminish the velocity and temperature profiles

iv. The heat and mass transfer and Sherwood number distributions are increasing significantly for the rise of Soret number

v. The rise of Schmidt number results in decrease of the heat and mass transfer and Sherwood number

vi. The shear stress decreases for increasing porous permeability parameter but an increase in the mass Grashof number is to increase the shear stress 


\section{References}

[1] A Barletta, S Lazzari, E Magyari and I Pop, Mixed convection with heating effects in a vertical porous annulus with a radially varying magnetic field, Int. J. Heat and Mass Transfer, vol. 51, pp. 5777-5784, 2008.

[2] A A Afify, Similarity solution in MHD effects of thermal diffusion and diffusion thermo on free convective heat and mass transfer over a stretching surface considering suction or injection, Communications in Nonlinear Science and Numerical Simulation, vol. 14, pp. 2202-2214, 2009.

[3] K A Yih, The effect of transpiration on coupled heat and mass transfer in mixed convection over a vertical plate embedded in a saturated porous medium, Int. Communications in Heat and Mass Transfer, vol. 24, pp. 265275, 1997.

[4] A Barletle, Combined forced and free convection with viscous dissipation in a vertical duct, Int. J. Heat and Mass Transfer, vol. 42, pp. 2243-2253, 1999.

[5] K E Chin, R Nazar, N M Arifin and I Pop, Effect of variable viscosity on mixed convection boundary layer flow over a vertical surface embedded in a porous medium, Int. Communications in Heat and Mass Transfer, vol. 34, pp. 464-473, 2007.

[6] S S Motsa, The effects of thermal radiation, hall currents, soret, and dufour on MHD flow by mixed convection over a vertical surface in porous media, SAMSA J. Pure and Applied Mathematics, vol. 3, 58-65, 2008.

[7] D Pal and B Talukdar, Perturbation analysis of unsteady magnetohydrodynamic convective heat and mass transfer in a boundary layer slip flow past a vertical permeable plate with thermal radiation and chemical reaction, Commun. Nonlinear Science and Numerical Simulation, vol. 15, pp. 1813-1830, 2010.

[8] N G Kafoussias and E W Williams, Thermal-diffusion and diffusionthermo effects on mixed free forced convective and mass transfer boundary layer flow with temperature dependent viscosity, Int. J. Engineering Science, vol. 33, pp. 1369-1384, 1995.

[9] M Angel, H S Takhar and I Pop, Dofour and Soret effects on free convection boundary layer over a vertical surface embedded in a porous medium, Studia universities- Bolyai, Mathematica, vol. 45, pp. 11-21, 2000.

[10] M S Alam and M M Rahman, Dufour and Soret effects on mixed convection flow past a vertical porous flat plate with variable suction, 
Nonlinear Analysis: Modelling and Control, vol. 11, pp. 3-12, 2006.

[11] R A Abreu, M F Alfradique and A S Telles, Boundary layer flows with Dufour and Soret effects: I: Forced and natural convection, Chemical Engineering Science, vol. 61, pp. 4282-4289, 2006.

[12] S N Gaikwad, M S Malashetty and K Rama-Prasad, An analytical study of linear and non-linear double diffusive convection with Soret and Dufour effects in couple stress fluid, Int. J. Non-Linear Mechanics, vol. 42, pp. 903-913, 2007.

[13] S El-Alimi, J Orfi and S Ben Nasrallah, Buoyancy effects on mixed convection heat and mass transfer in a duct with sudden expansions, Heat Mass Transfer, vol. 41, pp. 559-567, 2005.

[14] S Shateyi, Thermal radiation and buoyancy effects on heat and mass transfer over a semi-infinite stretching surface with suction and blowing, J. Applied Mathematics, Article ID 414830, 2008.

[15] A S Elzubier, S Y C Thomas, S Y Sergie, N L Chin and O M Ibrahim, The effect of buoyancy force in computational fluid dynamics simulation of a two-dimensional continuous ohmic heating process, American Journal of Applied Sciences, vol. 6, pp. 1902-1908, 2009.

[16] E M Abo-Eldahab and M Abd El-Aziz, Hall current and Ohmic heating effects on mixed convection boundary layer flow of a micropolar fluid from a rotating cone with power-law variation in surface temperature, Int. Communications Heat Mass Transfer, vol. 31, pp. 751-62, 2004.

[17] J S Osalusi and R Harris, Thermal-diffusion and diffusion-thermo effects on combined heat and mass transfer of a steady MHD convective and slip flow due to a rotating disk with viscous dissipation and Ohmic heating, Int. Communications in Heat and Mass Transfer, vol. 35, pp. 908915, 2008.

[18] D Pal and B Talukdar, Buoyancy and chemical reaction effects on MHD mixed convection heat and mass transfer in a porous medium with thermal radiation and Ohmic heating, Communications in Nonlinear Science and Numerical Simulation, vol. 15, pp. 2878-2893, 2010. 


\section{Appendix}

$$
\begin{gathered}
\beta_{1}=\frac{\operatorname{Pr}+\sqrt{\operatorname{Pr}^{2}+4 \operatorname{Pr} \alpha}}{2}, \beta_{2}=S c, \beta_{3}=\frac{1+\sqrt{1+4 M}}{2}, A_{1}=\frac{\operatorname{Sr} \beta_{1}}{\beta_{2}-\beta_{1}}, \\
A_{2}=\left(1-A_{1}\right), \\
A_{3}=\frac{-\left(A_{1} G r+G c\right)}{\beta_{1}^{2}-\beta_{1}-M^{2}}, A_{4}=\frac{-A_{2} G r}{\beta_{2}^{2}-\beta_{2}-M^{2}}, A_{5}=\frac{A}{M^{2}}, \\
A_{6}=\frac{-\left[A_{3}+A_{4}+A_{5}+\gamma\left(A_{3} \beta_{1}+A_{4} \beta_{2}\right)\right]}{1+\gamma \beta_{3}}, \\
A_{8}=\frac{-2 A_{4} A_{5} \operatorname{Pr} M^{2}}{\beta_{2}^{2}-\operatorname{Pr} \beta_{2}-\operatorname{Pr} \alpha}, A_{9}=\frac{-2 A_{5} A_{6} \operatorname{Pr} M^{2}}{\beta_{3}^{2}-\operatorname{Pr} \beta_{3}-\operatorname{Pr} \alpha}, \\
A_{11}=\frac{-2 A_{3} A_{5} \operatorname{Pr} M^{2}}{4 \beta_{2}^{2}-2 \operatorname{Pr} \beta_{2}-\operatorname{Pr} \alpha}, A_{12}=\frac{-A_{6}^{2} \operatorname{Pr}\left(\beta_{3}^{2}+M^{2}\right)}{4 \beta_{3}^{2}-2 \operatorname{Pr} \beta_{3}-\operatorname{Pr} \alpha}, \\
A_{10}=\frac{-A_{3}^{2} \operatorname{Pr}\left(\beta_{1}^{2}+M^{2}\right)}{4 \beta_{1}^{2}-2 \operatorname{Pr} \beta_{1}-\operatorname{Pr} \alpha}, \\
A_{15}=\frac{-2 A_{3} A_{4} \operatorname{Pr}\left(\beta_{1} \beta_{2}+M^{2}\right)}{\left(\beta_{1}+\beta_{2}\right)^{2}-\operatorname{Pr}\left(\beta_{1}+\beta_{2}\right)-\operatorname{Pr} \alpha}, \\
\frac{-2 A_{3} A_{6} \operatorname{Pr}\left(\beta_{1} \beta_{3}+M_{3}^{2}\right)}{\left(\beta_{2}\right)^{2}-\operatorname{Pr}\left(\beta_{1}+\beta_{3}\right)-\operatorname{Pr} \alpha}, A_{16}=\frac{A_{5}^{2} M^{2}}{\alpha}, \\
A_{14}{ }^{2}-\operatorname{Pr}\left(\beta_{2} \beta_{3}+M^{2}\right)
\end{gathered},
$$




$$
\begin{aligned}
& A_{17}=-\left(A_{7}+A_{8}+A_{9}+\ldots .+A_{16}\right), A_{18}=\left(A_{7}+A_{17}\right), A_{19}=\frac{-A_{18} S r \beta_{1}^{2}}{\beta_{1}^{2}-S c \beta_{1}}, \\
& A_{20}=\frac{-A_{8} S r \beta_{2}^{2}}{\beta_{2}^{2}-S c \beta_{2}} \\
& A_{21}=\frac{-A_{9} S r \beta_{3}^{2}}{\beta_{3}^{2}-S c \beta_{3}}, A_{22}=\frac{-4 A_{10} S r \beta_{1}^{2}}{4 \beta_{1}^{2}-2 S c \beta_{1}}, A_{23}=\frac{-4 A_{11} S r \beta_{2}^{2}}{4 \beta_{2}^{2}-2 S c \beta_{2}}, \\
& A_{24}=\frac{-4 A_{12} S r \beta_{3}^{2}}{4 \beta_{3}^{2}-2 S c \beta_{3}}, \\
& A_{25}=\frac{-A_{13} \operatorname{Sr}\left(\beta_{1}+\beta_{2}\right)^{2}}{\left(\beta_{1}+\beta_{2}\right)^{2}-S c\left(\beta_{1}+\beta_{2}\right)}, A_{26}=\frac{-A_{14} \operatorname{Sr}\left(\beta_{2}+\beta_{3}\right)^{2}}{\left(\beta_{2}+\beta_{3}\right)^{2}-S c\left(\beta_{2}+\beta_{3}\right)}, \\
& A_{27}=\frac{-A_{15} \operatorname{Sr}\left(\beta_{1}+\beta_{3}\right)^{2}}{\left(\beta_{1}+\beta_{3}\right)^{2}-S c\left(\beta_{1}+\beta_{3}\right)}, \\
& A_{28}=-\left(A_{19}+A_{20}+A_{21} \ldots .+A_{27}\right), A_{29}=\left(A_{20}+A_{28}\right) \text {, } \\
& A_{30}=\frac{-\left(A_{18} G r+A_{19} G c\right)}{\beta_{1}^{2}-\beta_{1}-M^{2}}, \\
& A_{31}=\frac{-\left(A_{8} G r+A_{29} G c\right)}{\beta_{2}^{2}-\beta_{2}-M^{2}}, A_{32}=\frac{-\left(A_{9} G r+A_{21} G c\right)}{\beta_{3}^{2}-\beta_{3}-M^{2}}, \\
& A_{33}=\frac{-\left(A_{10} G r+A_{22} G c\right)}{4 \beta_{1}^{2}-2 \beta_{1}-M^{2}}, \\
& A_{34}=\frac{-\left(A_{11} G r+A_{23} G c\right)}{4 \beta_{2}^{2}-2 \beta_{2}-M^{2}}, A_{35}=\frac{-\left(A_{12} G r+A_{24} G c\right)}{4 \beta_{3}^{2}-2 \beta_{3}-M^{2}}, \\
& A_{36}=\frac{-\left(A_{13} G r+A_{25} G c\right)^{2}}{\left(\beta_{1}+\beta_{2}\right)^{2}-\left(\beta_{1}+\beta_{2}\right)-M^{2}},
\end{aligned}
$$




$$
\begin{gathered}
A_{37}=\frac{-\left(A_{14} G r+A_{26} G c\right)^{2}}{\left(\beta_{2}+\beta_{3}\right)^{2}-\left(\beta_{2}+\beta_{3}\right)-M^{2}}, A_{38}=\frac{-\left(A_{15} G r+A_{27} G c\right)^{2}}{\left(\beta_{1}+\beta_{3}\right)^{2}-\left(\beta_{1}+\beta_{3}\right)-M^{2}} \\
A_{39}=\frac{A_{16} G r}{M^{2}}, \\
A_{40}=\frac{-1}{1+\gamma \beta_{3}}\left[A_{30}+A_{31}+A_{32}+\ldots .+A_{39}\right] \\
+\gamma\left[\begin{array}{l}
A_{30} \beta_{1}+A_{31} \beta_{2}+A_{32} \beta_{3} \\
+2 A_{33} \beta_{1}+2 A_{34} \beta_{2}+2 A_{35} \beta_{3} \\
+A_{36}\left(\beta_{1}+\beta_{2}\right)+A_{37}\left(\beta_{2}+\beta_{3}\right)+A_{38}\left(\beta_{1}+\beta_{3}\right)
\end{array}\right] \\
A_{41}=\left(A_{32}+A_{40}\right) .
\end{gathered}
$$

